\title{
Successful Management of a Widespread Osteosarcoma
}

\author{
A Case Report \\ Pentti Grohn ${ }^{a}$ Ashok Uttamchandani ${ }^{b}$ Zuhair Al-Haider ${ }^{b}$ Bruce Adams ${ }^{b}$ \\ Departments of a Nuclear Medicine and b Oncology, Mafraq Hospital, Abu Dhabi, United Arab Emirates
}

\section{Key Words}

Widespread osteosarcoma - Chemotherapy of osteosarcoma - Bisphosphonates · Increased survival

\begin{abstract}
Objective and Importance: To report the case of a 23year-old woman with widespread osteosarcoma including skeletal, pulmonary and pleural metastases, who had a remarkable response to combined chemo- and radiotherapy. Clinical Presentation and Intervention: A 23year-old Indonesian woman presented in October 1999 with a swelling of the right thigh, severe generalized pain and progressive left hemiparesis. Radiological examination revealed osteolytic lesions in the cervical spine. CT scan of the chest showed multiple pulmonary metastases and a huge left pleural effusion. Bone scan with technetium-99m hydroxymethylene diphosphonate showed intense uptake of the radiopharmaceutical in the distal right femur, generalized deposits throughout the skeleton and in the right hemithorax corresponding to the lung findings. Bone marrow and kidney function tests as well as serum calcium level were normal. Alkaline phosphatase was markedly elevated, 8,000 IU/I (nor$\mathrm{mal}<250 \mathrm{IU} / \mathrm{I})$. Histopathology from the femoral tumor showed osteosarcoma. Treatment was started with ra-
\end{abstract}

\section{KARGER}

Fax + 41613061234

E-Mail karger@karger.ch

www.karger.com
(C) 2004 S. Karger AG, Basel

1011-7571/04/0131-0054\$21.00/0

Accessible online at:

www. karger.com/mpp diotherapy to the cervical spine followed immediately by a combination chemotherapy with ifosfamide, cisplatin, etoposide and mesna rescue. In addition, the patient received bisphosphonates regularly. Eleven cycles of chemotherapy were given with a remarkable response. Conclusion: The patient was successfully treated with a combination of radio- and chemotherapy. She recovered fully and is in almost complete remission. The disease remained stable 24 months after the discontinuation of the treatment.

Copyright @ $(2004$ S. Karger AG, Base

\section{Introduction}

Malignant tumors arising from the skeletal system are rare and represent only $0.2 \%$ of all new cancers [1]. The most common bone tumors, osteosarcoma and Ewing's sarcoma, occur mainly in children and adolescents.

Widespread osteosarcoma is very difficult to treat. It rarely responds to cytotoxic chemotherapy and radiotherapy. We report the case of a 23-year-old woman with widespread osteosarcoma including skeletal, pulmonary and pleural metastases, who had a remarkable response to combined radio- and chemotherapy and has remained well 28 months after initial presentation. 
Fig. 1. Serial bone images before (a) and after (b) therapy.
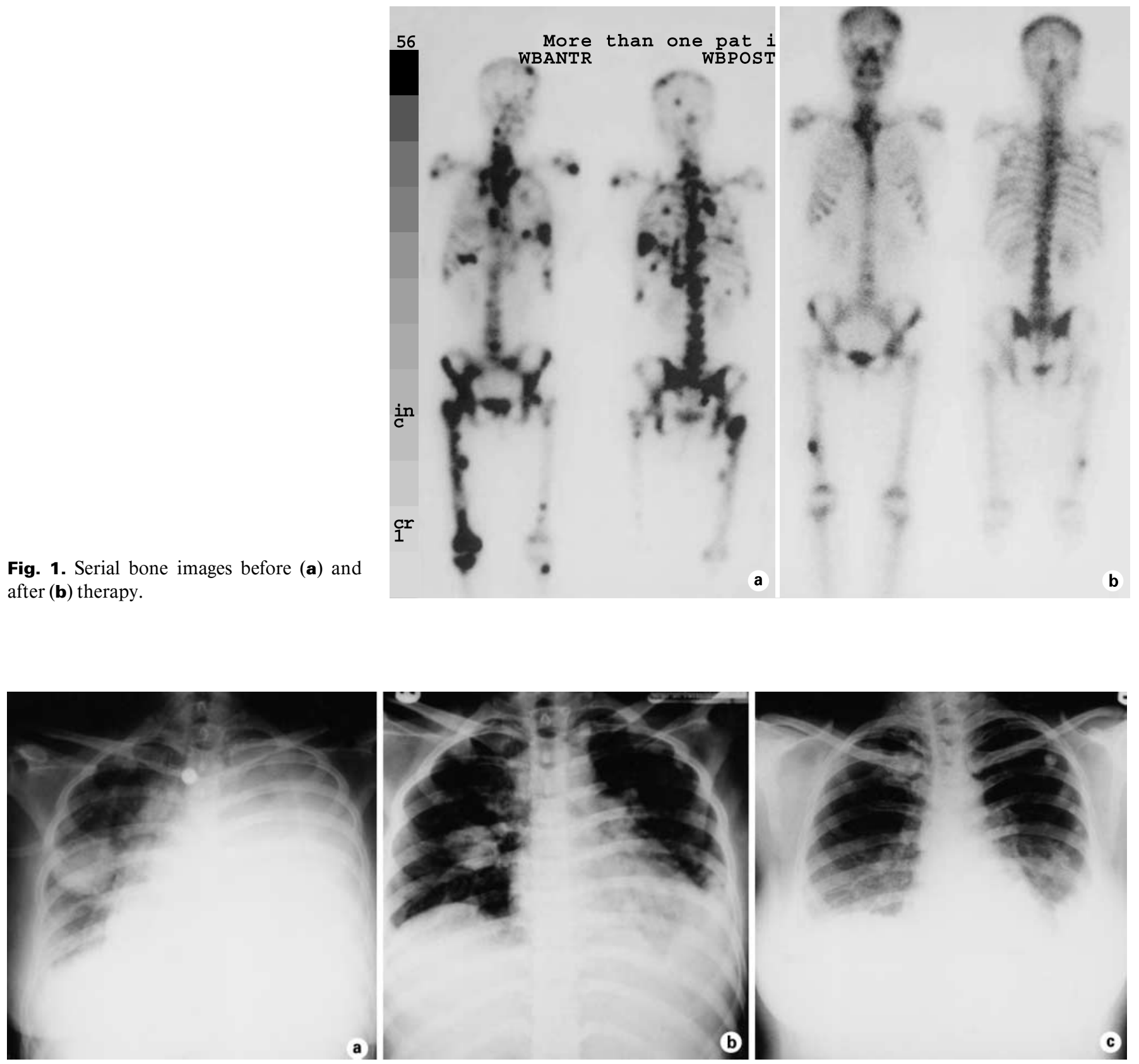

Fig. 2. Improvement of the pulmonary lesions seen on repeat chest radiographs.

\section{Case Report}

A 23-year-old woman presented in October 1999 with swelling of the right thigh, left hemiparesis and dyspnea, severe generalized pain especially in the neck and right femur. The symptoms progressed over 3.5 months. Bone radiographs showed osteolytic lesions from $\mathrm{C} 1$ to $\mathrm{T} 1$ and a big osteolytic mass lesion in the lower third of the femur. Chest radiography showed a cannon ball lesion on the right and a large pleural effusion on the left. CT scan of the chest showed multiple pulmonary metastases. Cytology from the aspirates revealed malignant cells. Bone marrow, kidney function as well as serum calcium level were normal. Alkaline phosphatase was markedly elevated, measuring $8,000 \mathrm{IU} / 1$ (normal $<250 \mathrm{IU} / 1$ ). Bone scan with technetium-99m hydroxymethylene diphosphonate showed intense radiopharmaceutical uptake in the distal femur, multiple deposits throughout the skeleton and in the right hemithorax corresponding to a pulmonary deposit. Histopathology of the femoral tumor indicated an osteosarcoma. 
Initial treatment was radiotherapy to the cervical spine. A total dose of 30 Gy was administered in 10 equal fractions over a 10-day period. Chemotherapy was started immediately after the radiotherapy, in November 1999. The regimen consisted of ifosfamide $\left(1,200 \mathrm{mg} / \mathrm{m}^{2}\right.$ i.v. on days $1-3$ with mesna rescue), cisplatin $(60 \mathrm{mg} /$ $\mathrm{m}^{2}$ i.v. on day 1$)$ and etoposide $\left(100 \mathrm{mg} / \mathrm{m}^{2}\right.$ i.v. on days $\left.1-3\right)$ given in all cycles every 21 days.

The response to the initial radiotherapy was positive, the hemiparesis started to disappear. The response to the chemotherapy was remarkable. After 2 cycles the patient was almost totally free of body pain and could be discharged from the hospital. The remaining 9 cycles of chemotherapy were continued on an ambulatory basis. The last was given in August 2000. In addition to chemotherapy, the patient received monthly infusion bisphosphonate (Bonefos or Aredia). The patient's response to therapy was monitored with bone scintigraphy; 7 bone scans were performed over a period of 2 years following her initial admission. She demonstrated a dramatic improvement that included a marked reduction in the number of metastatic lesions as well as in the intensity of the uptake of the radiopharmaceutical (fig. 1). The patient experienced an excellent subjective response to therapy and good recovery based on objective clinical criteria as evidenced on the bone scans (fig. 1) and chest radiographs (fig. 2). The smaller pulmonary lesion had disappeared and the larger ones had become densely calcified masses (fig. 2). Alkaline phosphatase returned to normal level. A biopsy taken from the primary site of the tumor showed no malignancy. During the 24-month follow-up after the discontinuation of chemotherapy, the disease has remained stable. At the moment the patient is continuing her daily work and routine activities.

\section{Discussion}

The treatment of primary metastatic widespread osteosarcoma is difficult. Amputation of the limb or wide excision of the tumor is the standard treatment regime. The prognosis after surgery alone is poor because the patient generally develops lung metastases within 6 months and 5-year survival is only 15-20\% [2]. Over the past two decades the introduction of limb-sparing surgery with concomitant neoadjuvant and adjuvant chemothera- py has changed the prognosis dramatically, resulting in a 5-year survival of 55-80\% in nonmetastatic patients [3]. Multiple drug regimens with surgery are now considered essential treatment.

The response rate to single-drug treatment of metastatic osteosarcoma is not very good, hence a combination of drugs is used (doxorubicin, platins, ifosfamide and highdose methotrexate) with response rates of $25-40 \%$ [4]. In addition, bisphosphonates and analgesics are given for pain palliation, the former possibly potentiates the response to chemotherapy, and at least enhances the recalcification of the lytic bone lesions and prevents hypercalcemia.

The treatment of metastatic osteosarcoma is based on combination chemotherapy, bisphosphonates and pain control with radiotherapy and analgesics. Complete remissions are rare, partial responses occur in less than half of the patients. Median survival is approximately 6 months [5].

The role of bisphosphonates in the treatment of osteosarcoma is not known. They may act by recalcifying osteolytic lesions, reducing pain and preventing hypercalcemia. Surgery was not considered because of the multiple lesions. The patient is now in almost complete remission, her symptoms are stable and she is back to her daily work. She is totally functional 24 months after the discontinuation of therapy.

\section{Conclusion}

The patient with metastatic osteosarcoma, treated with a combination of radio- and chemotherapy as well as bisphosphonates, had an excellent clinical outcome. She is in good condition and has returned to her daily activities 24 months after the discontinuation of the therapy.

\section{References}

1 Mankin $\mathrm{H}$, Willet $\mathrm{C}$, Harmon D: Malignant tumours of the bone; in Holleb A, Fink D, Murphy G (eds): American Cancer Society Textbook of Oncology. 1994, pp 355-358.

2 Ward M, Mikaelian K, Dorey F: Pulmonary metastases in stage IIB extremity osteosarcoma. J Clin Oncol 1994;12:1849-1858.

3 Bacci G, Ferrari S, Bertoni F, Ruggieri P: Longterm outcome for patients with non-metastatic osteosarcoma of extremity treated at the Instituto Ortopedico Rizzoli according to the Instituto Ortopedico Rizzoli/Osteosarcoma-2 protocol: An updated report. J Clin Oncol 2000; 18:4016-4027.
4 Provisor A, Ettinger L, Nachman J: Treatment of nonmetastatic osteosarcoma of the extremity with preoperative and postoperative chemotherapy. J Clin Oncol 1997; 15:74-78.

5 Ferrari S, Bacci G, Picci P: Long-term followup and postrelapse survival in patients with nonmetastatic osteosarcoma of the extremity treated with neo-adjuvant chemotherapy. Ann Oncol 1997;8:767-771. 